Chinese Journal of Organic Chemistry

ACCOUNT

\title{
芳基高价碘盐诱导的亲电环化反应研究
}

\author{
陈 静 $a, b$ 曲红梅*,a 彭 静 $b$ 陈 超 ${ }^{*}, b$ \\ ( ${ }^{a}$ 天津大学化工学院 系统生物工程教育部重点实验室 天津市生物制药工程重点实验室 天津 300072) \\ ( ${ }^{b}$ 清华大学化学系 生命有机磷化学及化学生物学教育部重点实验室 北京 100084)
}

\begin{abstract}
摘要 亲电试剂诱导的环化反应是构建环状化合物的有效策略, 而利用芳基高价碘盐作为亲电试剂诱导环化反应已经 取得了诸多研究进展. 在这些亲电环化反应中, 高价碘盐不仅可以作为芳基化试剂诱导环化反应形成芳基取代环化物, 还可以诱导苯并环化反应, 形成苯并环状化合物.

关键词 芳基高价碘; 亲电环化; 芳化-环化反应; 苯并环化反应
\end{abstract}

\section{Study of Electrophilic Cyclization Reactions Triggered by Diaryliodonium Salts}

\author{
Chen, Jing ${ }^{a, b} \quad$ Qu, Hongmei ${ }^{*, a} \quad$ Peng, Jing ${ }^{b} \quad$ Chen, Chao ${ }^{*, b}$
}

( ${ }^{a}$ Key Laboratory of Systems Bioengineering, Ministry of Education, Tianjin Key Laboratory of Biological and Pharmaceutical Engineering, School of Chemical Engineering and Technology, Tianjin University, Tianjin 300072)

$\left({ }^{b}\right.$ Key Laboratory of Bioorganic Phosphorus Chemistry \& Chemical Biology, Ministry of Education, Department of Chemistry, Tsinghua University, Beijing 100084)

\begin{abstract}
Electrophilic cyclization reactions are one of the most useful strategies for the construction of various cyclic compounds and significant progress has been made in the electrophilic cyclizations triggered by diaryliodonium salts. In these new types of electrophilic cyclization reactions, diaryliodonium salts can not only act as arylation reagents to initiate arylation-cyclization reactions giving aryl-substituted compounds, but also to be involved in the benzocyclization to give benzocycles.

Keywords diaryliodonium salts; electrophilic cyclization; arylation-cyclization reactions; benzocyclization
\end{abstract}

芳基高价碘盐 $\mathrm{Ar}_{2} \mathrm{I}^{+} \mathrm{X}^{-}$是一种高亲电性的芳基化试 剂 ${ }^{[1]}$. 这类试剂具有环境友好、性质稳定、反应选择性 高的优点, 被广泛应用于现代有机合成化学中 ${ }^{[2]}$. 近年 来, Olofsson 课题组和 Macmillan 课题组 ${ }^{[3]}$ 的研究为芳基 高价碘盐的制备提供了更简单、更可靠的方法，进一步 推动了高价碘试剂在有机合成领域中的应用. 如 Scheme 1 所示, 这类试剂很容易由芳基碘或芳烃-碘单 质制备, 一般一锅法经两到三步即可得到.

通常, 芳基高价碘盐具有很高的亲电性, 可以看成 芳基正离子的等价体, 与亲核试剂反应形成单取代的芳 基化物(Scheme 2, a $)^{[4]}$, 但很少有文献报道芳基高价碘 试剂作为碳基亲电体诱导的环化反应 ${ }^{[5]}$. 我们课题组通
过近年的研究, 发现了一系列高价碘试剂诱导的亲电环 化反应(Scheme 2, b)，其中芳基高价碘可以作为芳基化 试剂提供芳基基团诱导环化反应的发生. 此外, 进一步 研究发现芳基高价碘还可以诱导苯并环化反应的发生, 合成一系列复杂的苯并环化产物.

\section{1 芳基高价碘试剂诱导的单环化反应形成单环 化合物}

\section{1 芳基高价碘试剂促进的炔烃芳基-烷基化反应}

在不活泼的 $\mathrm{C}-\mathrm{H}$ 键上形成 $\mathrm{C}-\mathrm{C}$ 键来构建复杂化 合物的合成策略具有步骤简单、原子经济性高的优点 ${ }^{[6]}$,

\footnotetext{
*E-mail: ququhongmei@126.com; chenchao01@mails.tsinghua.edu.cn Received January 5, 2015; revised January 30, 2015; published online February 10, 2015

Project supported by the National Natural Science Foundation of China (Nos. 21102099, 21102080, 21372138) and the Tsinghua University Initiative Scientific Research Program (No. 2011Z02150).

国家自然科学基金(Nos. 21102099, 21102080, 21372138)和教育部-清华大学自主科研计划(No. 2011Z02150)资助项目.
} 
(a)

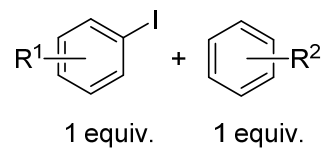

(1) $m$-CPBA (1 equiv.)

(2) $\mathrm{TfOH}(2 \sim 3$ equiv. $)$

$\mathrm{CH}_{2} \mathrm{Cl}_{2}$, r.t., ca. $1 \mathrm{~h}$

$51 \% \sim 92 \%$<smiles>[R17]c1ccc([I-][Te]c2ccccc2)cc1</smiles><smiles>[R]c1ccc([I-]([O])c2ccc([R])cc2)cc1</smiles>

(b)

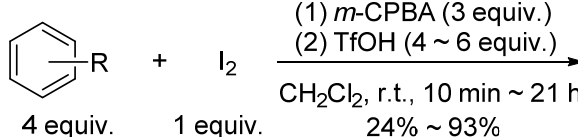

(1) $m$-CPBA ( 3 equiv.) $24 \% \sim 93 \%$

(c)

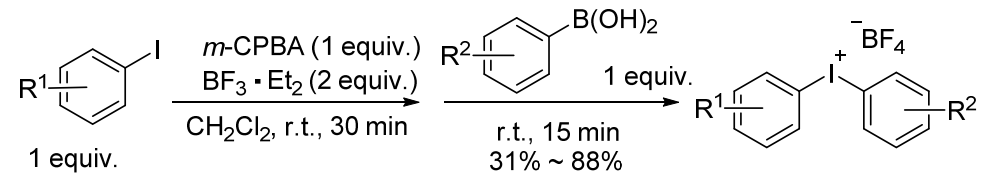

图式 1 一锅法制备二芳基高价碘盐

Scheme 1 One-pot syntheses of diaryliodonium salts

(a) ${ }^{\mathrm{R}}$<smiles>[R]c1ccc([I-]c2ccc(CC(C)C)c([R])c2)cc1</smiles><smiles>N#Cc1ccc(N)cc1</smiles>

(b)

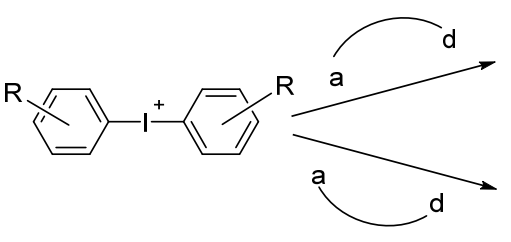<smiles>c1ccc(B2CCCCC2)cc1</smiles>
Arylation-cyclization

图式 2 高价碘试剂诱导的亲电环化反应

Scheme 2 Electrophilic cyclization reactions triggered by diaryliodonium salts

但大多数研究集中于 $\mathrm{C}_{\mathrm{sp}^{2}}-\mathrm{H}$ 活化, 而相对反应活性更 低的 $\mathrm{C}_{\mathrm{sp}}{ }^{3}-\mathrm{H}$ 键活化研究较少, 且一般需要合适的杂原 子(如 $\mathrm{N}$ 原子或 $\mathrm{O}$ 原子)、诱导基团、或者是稀有金属的 参与 ${ }^{[7]}$. 我们课题组 ${ }^{[8]}$ 的研究发现, 在铜盐催化下, 芳基 高价磑试剂与炔烃能够发生芳基-烷基化反应. 反应过 程中, 不活泼的烷基 $\mathrm{C}_{\mathrm{sp}^{3}}-\mathrm{H}$ 键上可形成新的 $\mathrm{C}-\mathrm{C}$ 键, 高价碘试剂提供芳基, 诱导环化反应的发生形成单环化 合物, 芳环上的一个 $\mathrm{C}$ 原子通过 $\mathrm{C}-\mathrm{C}$ 键与新形成的五 元环连接.

如 Eq. 1 所示, 在 $\mathrm{Cu}(\mathrm{OTf})_{2}$ (约 $5 \mathrm{~mol} \%$ ) 催化下, 二苯 基六氟磷酸高价碘(1a)与 1-苯基-1-已炔(2a)反应, 环化 作用发生在炔烃 2a 的 C(1) 和 C(5)位置, 形成新的 C-C 键, 得到具有单一位置选择性的芳基环化产物 3a. 为了 进一步扩展这种方法并体现其合成价值, 对不同取代基 团的炔烃(如延长烷基链长、炔烃端位由大位阻基团取 代、芳环上带有不同电性取代基团)进行了尝试, 而各种 对称或不对称结构的芳基高价碘试剂对反应的适应性 也得到了深入研究, 实验证明这类反应具有很好的官能
团容忍性及区域选择性(Scheme 3).

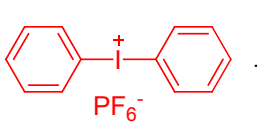

$1 \mathrm{a}$<smiles>CCCCC(C)CCC#Cc1ccccc1</smiles>

$2 a$

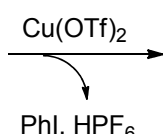

Phl, $\mathrm{HPF}_{6}$<smiles>C[C@H]1CCC(c2ccccc2)=C1c1ccccc1</smiles>

在以往研究中, 通过螺环中心形成 $\mathrm{C}-\mathrm{C}$ 键来构建 螺环化合物的方法一直较难实现, 究其原因在于官能化 后的季碳原子反应活性不稳定, 并且具有很大的位阻, 不利于反应顺利进行 ${ }^{[9]}$. 而通过芳基高价碘诱导亲电环 化反应的策略, 以 $2 \mathrm{~b}, 2 \mathrm{c}$ 为起始底物, 采用同样的铜盐 催化条件, 可以高选择性得到复杂螺环化合物产物 $\mathbf{3} \mathbf{j} \sim$ $3 \mathbf{m}$ ，极大简化了此类化合物的合成步骤( Scheme 3). 


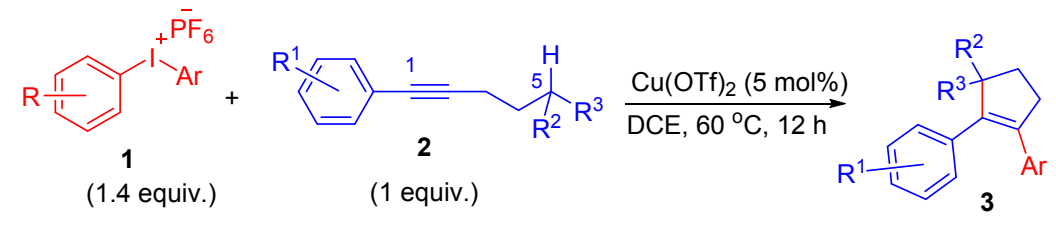

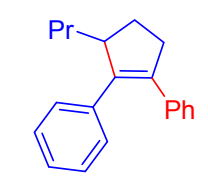

3b $71 \%$

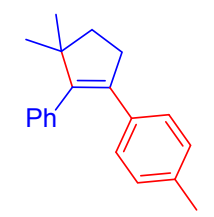

3f $90 \%$

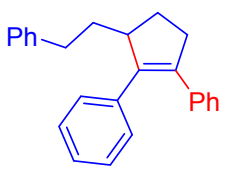

3c $60 \%$

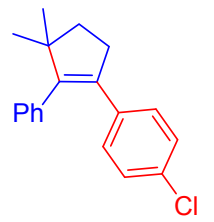

3 g $90 \%$

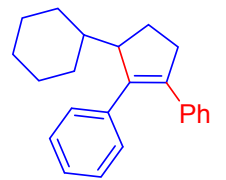

3d $70 \%$

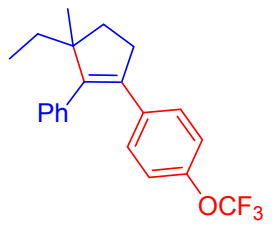

3h $51 \%$

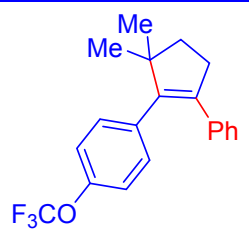

3e $72 \%$

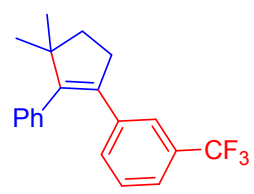

3i $65 \%$

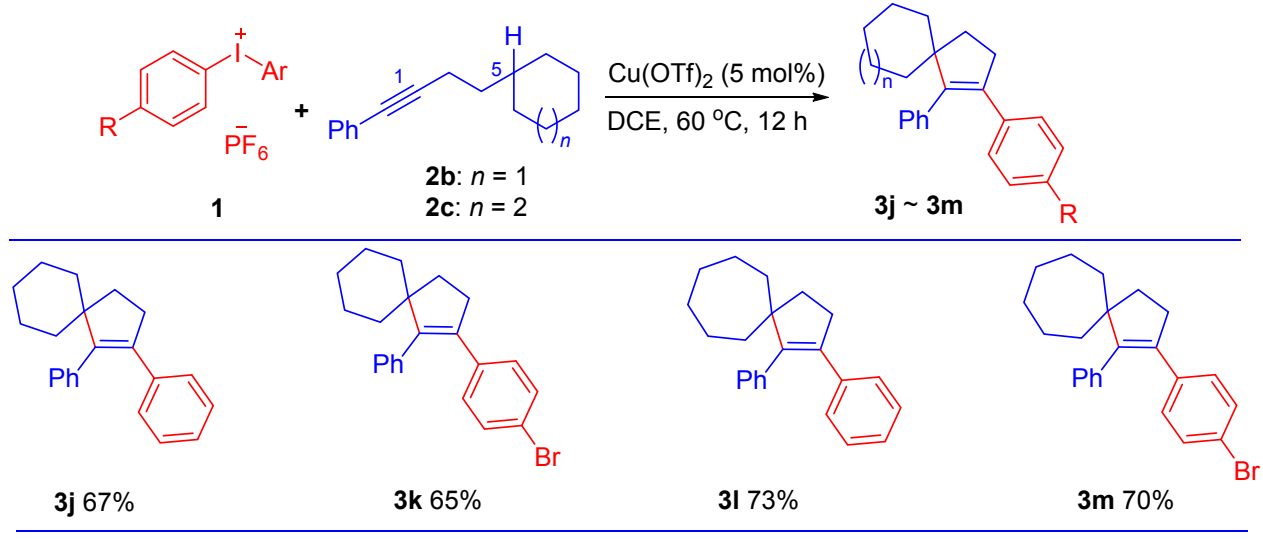

图式 3 芳基高价碘试剂促进的炔烃芳基-烷基化反应

Scheme 3 Aryl-carbocyclization of alkynes triggered by diaryliodonium salts

\section{2 芳基高价碘试剂促进的炔烃芳基-醚化反应}

炔烃的芳基-醚化反应是合成含氧杂环的重要方法, 这类反应往往需要钯催化剂来协助完成. 而我们的研究 发现, 烷氧基炔与芳基高价碘试剂的分子内芳醚化反应 在铜盐催化下就可以实现(Scheme 4) ${ }^{[10]}$. 在这个反应中, 采用简单易得具有线性结构的烷氧基炔烃分子为起始 原料, 在高价碘试剂及铜盐催化剂的诱导下, 通过亲电 环化作用使稳定的 $\mathrm{C}-\mathrm{O}$ 键断裂从而形成各种含氧杂环 分子 ${ }^{[1]}$. 这其中芳基高价碘亦作芳基化试剂提供芳环 基团, 诱导环化反应的发生. 如 Scheme 4 所示, 该方法 可以用于制备各种二芳基取代的含氧杂环(其中包括五 元杂环和六元杂环), 而具有类似结构的 2'-甲氧基二苯 乙炔(6)也可以与芳基高价碘反应, 产物为二芳基取代 的苯并呋喃 7 .

为了实现更好的应用, 我们对所得的含氧杂环化合 物进行进一步官能化, 如在 $\mathrm{NiCl}_{2}\left(\mathrm{PPh}_{3}\right)_{2}$ 催化下, 5 与格
氏试剂发生偶联作用可得到三苯基取代烯醇 $\mathbf{8}$, 此种烯 醇是抗癌药物他莫西芬的重要代谢中间体 ${ }^{[12]}$.

研究表明在铜盐的存在下, 高价碘试剂能够原位转 化生成具有高亲电性的三价芳基铜 $\mathrm{Ar}-\mathrm{Cu}(\mathrm{III}) \mathbf{I}^{[13]}$, 这 种三价芳基铜 $\mathbf{I}$ 很容易被炔基进攻产生烯铜结构的中间 体 II, 而中间体 II 与中间体 III 可互变. 当反应底物为 烷氧基取代的炔烃时，高电负性的 $\mathrm{O}$ 原子可以进攻 $\mathrm{Cu}$ 中心 (之后伴随 $\mathrm{Cu}(\mathrm{I}) \mathrm{X}$ 消除)或进攻烯基正得到氧鎓结构 的中间体 IV, 最终经取代作用得到芳基醚化成环产物 5. 为了验证反应机理, 以 $\mathrm{R}$ 为大取代基团的 $4 \mathrm{x}$ 作为起 始底物, 在标准条件下与芳基高价碘作用, GC-MS 检测 反应发现, 除了主要产物 5a, 还检测到一定量 3-(4-溴苯 基) -氟代丙烷和 4-烯丙基溴苯. 这一实验现象验证了反 应过程中氧鎓结构中间体 IV 的存在，整个过程，芳基 高价碘试剂提供单个芳基诱导了反应发生(Scheme 5). 


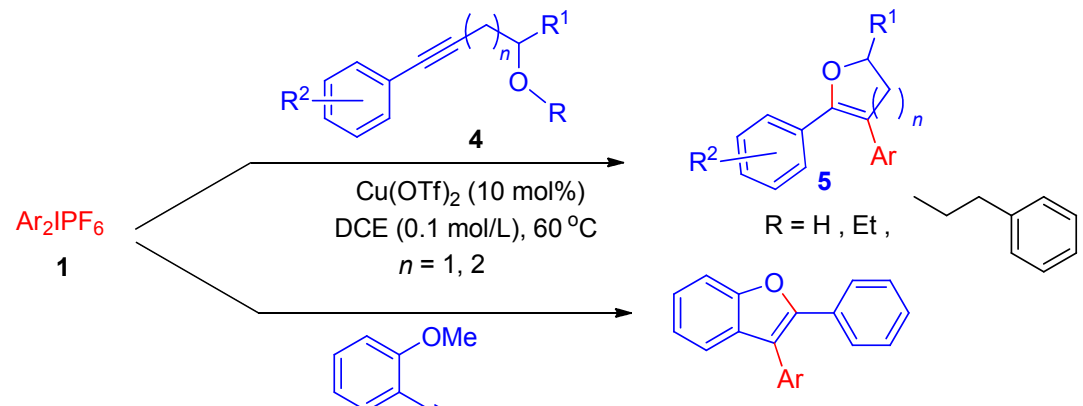

6

benzofuran

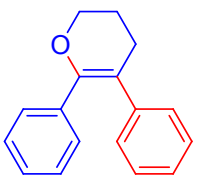

5a: $92 \%$

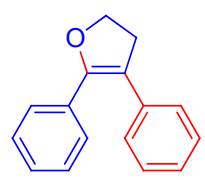

5e: $62 \%$

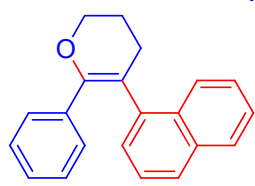

5b: $31 \%$

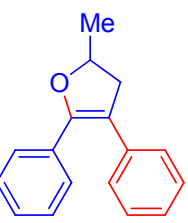

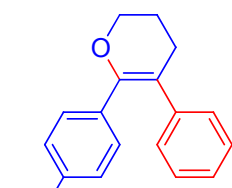

5c: $88 \%$

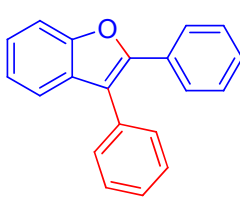

5g: $37 \%$

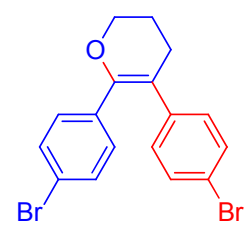

5d: $96 \%$

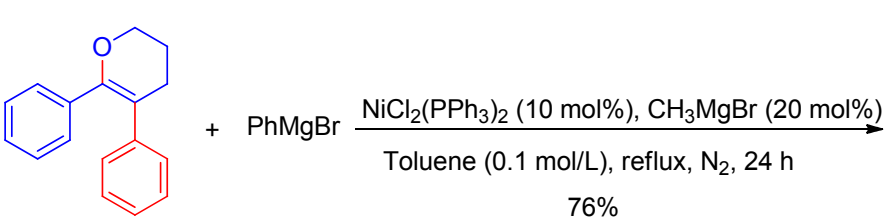

$5 a$ $76 \%$

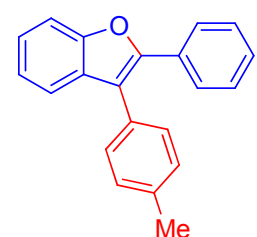

5h: $40 \%$

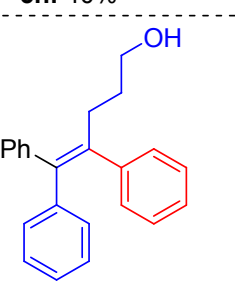

图式 4 芳基高价碘试剂促进的炔烃芳基-醚化反应

Scheme 4 Aryl-etherification of alkoxyl alkynes triggered by diaryliodonium salts

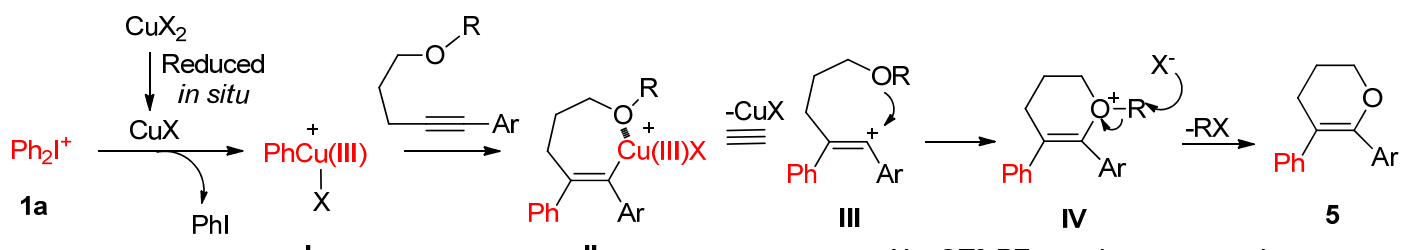

$$
\text { II }
$$

$\mathrm{X}=\mathrm{OTf}, \mathrm{PF}_{6}$ or other counteranion

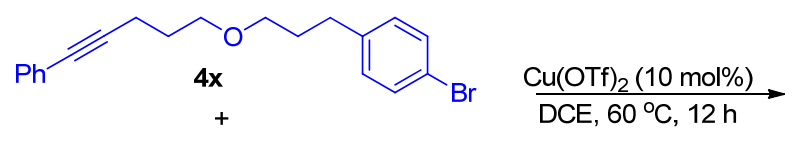

$\mathrm{Ph}_{2} \mathrm{IPF}_{6}$

via<smiles>[X]c1ccccc1</smiles>

\section{$5 \mathbf{a}$}

$85 \%$
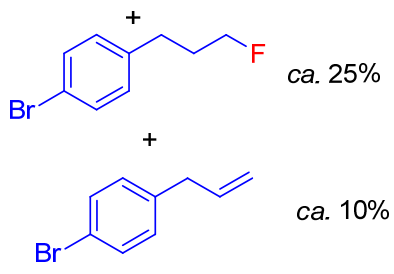

图式 5 分子内芳基-醚化反应机理

Scheme 5 Plausible mechanism for intramolecular aryl-etherification reaction 


\section{2 芳基高价碘试剂参与亲电环化反应形成苯并 环化物}

含 $\mathrm{N}$ 杂环化合物特别是喹啉及喹唑啉类衍生物是 许多生物活性物质的骨架结构, 在药物化学、农用化学、 功能化有机材料方面有广泛应用, 而制备多种基团取代 的喹啉及喹唑啉类衍生物一直是化学合成领域的热 点 ${ }^{[14]}$. 传统人名反应制备喹啉类衍生物的方法有 Skraup 合成法、Combes 合成法、Conrad-Limpach 合成法等, 而 制备喹唑啉类衍生物的方法有 Niementowski 合成法及 改进的 Duff 合成法. 但这些经典方法存在着底物毒性 大(基本都是苯胺衍生物)、反应条件苛刻(通过强酸、强 碱存在下的缩合反应完成)、官能团容忍度低等各种缺 陷. 发展高效便捷的杂环合成方法对于有机合成、药物 笁选和有机光电材料的发展都具有重要意义. 我们课题 组尝试利用芳基高价碘试剂的亲电环化作用来实现这 一类含 $\mathrm{N}$ 杂环化合物的制备, 获得了可喜的结果.

\section{1 高价碘试剂参与的喹啉合成法}

研究发现选择三种小分子物质: 二芳基高价碘、腈、 炔作为底物, 在催化量 $\mathrm{Cu}(\mathrm{OTf})_{2}$ 作用下即可有效合成带 有各种取代基团的喹啉衍生物 ${ }^{[15]}$. 反应过程中, 首先, 芳基高价碘试剂原位生成三价芳基铜 $\mathrm{Ar}-\mathrm{Cu}(\mathrm{III})$, 之后 $\mathrm{Ar}-\mathrm{Cu}$ (III) 将芳基基团转移至腈上得到 $N$-苯基乙腈结构 的中间体 $\mathbf{A}$, 此中间体 $\mathbf{A}$ 被炔基进攻得到中间体 $\mathbf{B}$ (电子
效应主导), 后经由亲电环化作用最终产生喹啉类衍生 物(Scheme 6). 反应过程中, 芳基高价碘诱导苯并环化 反应参与成环, 形成苯并杂环. 由于反应途径中多个碳 正离子中间体的存在，使得此反应具有很高的活性及立 体选择性. 这种 $[2+2+2]$ 模式三组份环化反应相对已 知缩合反应具有原料简单易得、反应条件温和、取代基 容忍度高、区域选择性好的特点, 为合成不同官能团取 代喹啉衍生物提供了一条有效途径.

\section{2 高价碘试剂参与的喹唑啉合成法}

进一步将 $[2+2+2]$ 夰联反应模式推广，应用到喹 唑啉衍生物的制备 ${ }^{[16]}$. 采用芳基高价碘试剂和两分子 腈作为底物, 铜盐催化下成功合成各种取代基取代的喹 唑啉衍生物. 芳基高价碘试剂作为亲电体诱导苯并环化 反应，形成苯并杂环. 而更加具有挑战性的问题是如何 采用不同腈类作为底物, 制备 $\mathrm{R}^{1}$ 与 $\mathrm{R}^{2}$ 不同的喹唑啉衍 生物. 最终, 我们通过控制加料时间, 避免底物相似性 带来的干扰，实现了一锅法制备不同基团官能化的喹唑 啉类产物(Scheme 7).

\section{3 高价磑试剂参与的多环氮杂环的合成}

多环喹啉类是许多天然产物和化学合成药物的骨 架结构, 例如: 天然拓扑异构酶 I 的抑制剂 luotonin A (1)、抗癌药物喜树碱(2)以及最早被发现可以用于治疗 阿尔兹海默症的胆碱酯酶抑制剂他可莫(商品名

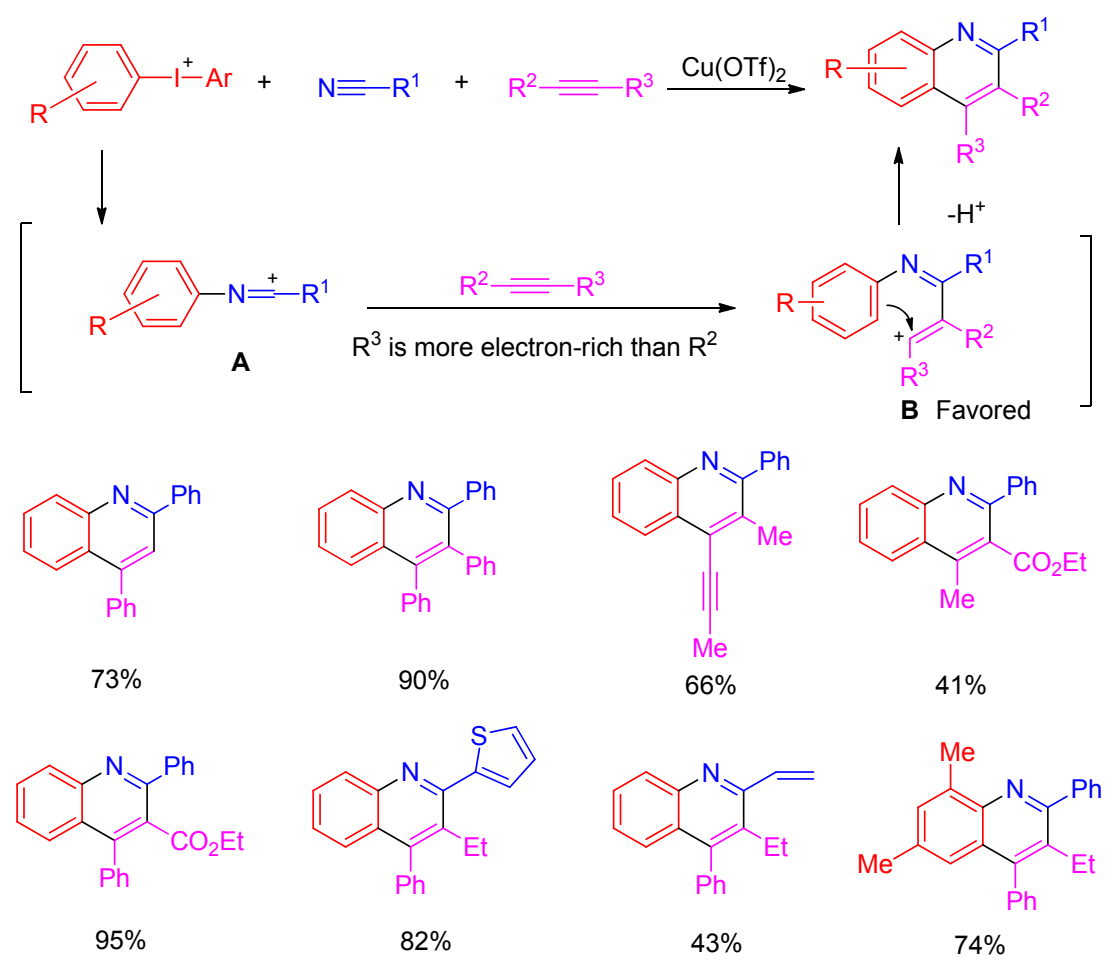

图式 6 高价碘参与的三组份串联环化反应制备喹啉衍生物

Scheme 6 Synthesis of quinolines by three-component cascade annulation with diaryliodonium salts 


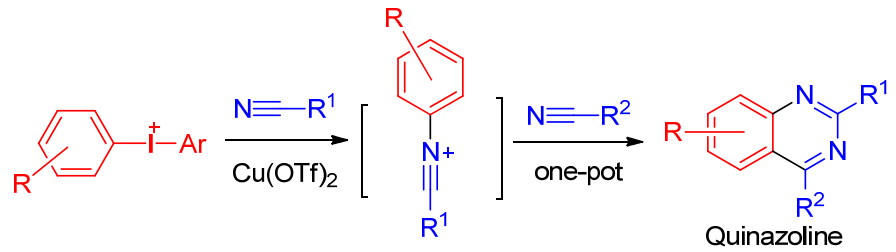<smiles>Brc1ccc2nc(-c3ccccc3)nc(-c3ccccc3)c2c1</smiles>

$72 \%$<smiles>O=C(c1ccccc1)c1nc(-c2ccccc2)nc2ccccc12</smiles>

$61 \%$<smiles>Cc1ccc2nc(-c3ccccc3)nc(-c3ccccc3)c2c1</smiles>

$89 \%$<smiles>Brc1ccccc1-c1nc(-c2cccs2)c2ccccc2n1</smiles>

$63 \%$<smiles>Cc1cc(C)c2nc(-c3ccccc3)nc(-c3ccccc3)c2c1</smiles>

$68 \%$<smiles>COc1ccc(-c2nc(Br)c3ccccc3n2)cc1</smiles><smiles>c1ccc(-c2nc(-c3ccccc3)c3ccc4ccccc4c3n2)cc1</smiles>

$66 \%$<smiles>CCOC(=O)c1nc(-c2ccc(OC)cc2)nc2ccccc12</smiles>

图式 7 高价碘参与的一锅法制备喹唑啉类衍生物

Scheme 7 One-pot synthesis of quinazolines from diaryliodonium salts

Cognex) (3) ${ }^{[17]}$. 因此, 发展此类多环含 $\mathrm{N}$ 化合物的制备 方法具有重要意义. 研究发现, 采用 $\omega$-氭基-1-炔 9 作为 反应底物, 在铜盐催化下与芳基高价碘试剂作用发生分 子内 $[2+2+2]$ 环加成反应, 可以得到相应多环喹啉结 构的产物 ${ }^{[18]}$. 如 Scheme 8 所示, 通过这种途径可以制 备各种多苯并环的喹啉衍生物. 而通过对具有 $\mathrm{Br}$ 原子
取代基的产物进行氨化我们成功制备了药物活性物质 他可林 ${ }^{[19]}$ ，更多具体官能化应用也已报道.

与之相似，当采用 $\omega$-叠氮-1-炔 10 作为底物时，高 亲电性的芳基高价碘试剂首先进攻叠氮基团，后参与苯 并环化过程，最终也可得到多环喹啉衍生物 ${ }^{[20]}$.

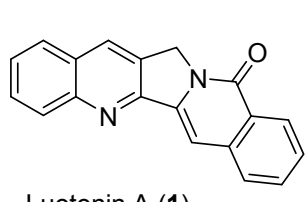

Luotonin A (1)<smiles>CC[C@@]1(O)C(=O)OCc2c1cc1n(c2=O)Cc2cc3ccccc3nc2-1</smiles>

Camptothecin (2)<smiles>Nc1c2c(nc3ccccc13)CCCC2</smiles>

Tacrine (3)<smiles>[R]c1cccc([I+][Al-])c1</smiles>

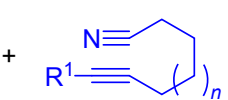<smiles>[R]c1ccc([I+][Al-])cc1</smiles>

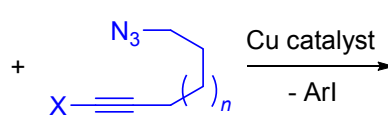

10<smiles>[R]c1ccc2cc3c(nc2c1)CCCC3</smiles>

$\mathrm{R}^{1} \quad \mathrm{R}=\mathrm{Ar}, \mathrm{Br}, \mathrm{Cl}$, etc. $n=0,1,2,3$<smiles>[X]c1c2c(nc3ccccc13)CCC2[R]</smiles><smiles>Nc1c2c(nc3ccccc13)CCCC2</smiles>
$\mathrm{Cu}_{2} \mathrm{O}(10 \mathrm{~mol} \%)$ NMP, $100^{\circ} \mathrm{C}, 2 \mathrm{~d}$ $69 \%$<smiles>Brc1c2c(nc3ccccc13)CCCC2</smiles>
$n=1,2,3$<smiles>Cc1cccc(-c2c3c(nc4ccccc24)CCCC3)c1</smiles>

$85^{\circ} \mathrm{C}, 12 \mathrm{~h}, 96 \%$

图式 8 高价碘参与的多环喹啉化合物制备

Scheme 8 Synthesis of polycyclic quinolines from diaryliodonium salts 


\section{4 高价碘试剂与 $O$-氭基苯胺的多样性反应: 喹唑啉 亚胺与苯胺基叫啶的合成}

在开展芳基高价碘试剂诱导 $o$-氰基苯胺 11 的亲电 环化制备含 $\mathrm{N}$ 化合物的研究中(Scheme 9), 当采用 2 equiv. $O$-氰基苯胺 $\mathbf{1 1}$ 作为底物时 ${ }^{[21]}$, 可以得到非常重要 的喹唑啉亚胺衍生物 12(其中芳基高价碘试剂作为芳基 化试剂诱导芳环化反应发生, Pathway A).

改变温度并采用 0.5 equiv. $O$-氰基苯胺和芳基高价 碘试剂反应, 还可以选择性获得在染料、药物、有机材 料方面有重要应用的鲐啶产物 $\mathbf{1 3}^{[22]}$, 而在这个反应过 程 Pathway B 中, 芳基高价碘试剂可同时诱导芳-环化 反应和苯并环化反应.

机理如 Scheme 10 所示: 首先, $O$-氰基苯胺 11 与芳 基高价碘 1 在催化剂 $\mathrm{Cu}(\mathrm{OTf})_{2}$ 诱导下产生 $N$-苯基腈鎓 离子 14, 当体系中存在过量 $o$-氰基苯胺 11 时, 氨基进 攻中间体 $N$-苯基腈鎓离子经中间体 $\mathbf{I}$ 发生串联环化作用
最终得到喹唑啉亚胺产物 12 (Pathway A).

另一方面, 当反应体系中存在过量高价碘试剂 $\mathbf{1}$ 时, 中间体 $N$-苯基腈鎓离子会发生进一步芳基化作用产生 中间体 II, 之后经分子内亲电取代产生吵啶结构产物 $\mathbf{1 3}$ (Pathway B). 值得指出的是, 这两种途径在不同温度下 进行，具有很高的选择性.

\section{3 结论与展望}

利用高亲电性的芳基高价碘盐，发展了一系列以简 单易得分子作为起始底物, 反应条件温和, 高选择性制 备复杂产物的亲电环化反应. 在这些合成策略中，芳基 高价碘试剂可作芳基化试剂诱导芳化反应发生，也可以 诱导苯并环化反应，或者两种反应途径兼备的方式完成 亲电环化过程. 从合成角度看, 芳基高价碘盐参与的亲 电环化反应克服了许多在经典反应过程中存在的困难,

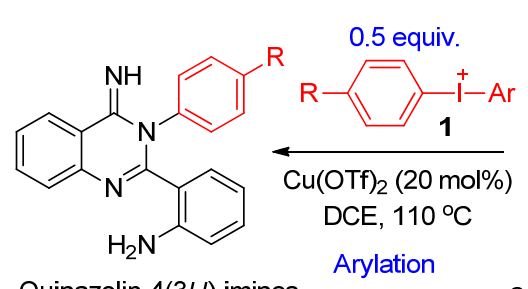

Quinazolin-4(3H)-imines

12<smiles>Cc1ccc(-n2c(-c3ccccc3N)nc3ccccc3c2=N)cc1</smiles>

12a, $95 \%$<smiles>N=c1c2ccccc2nc(-c2ccccc2N)n1-c1ccccc1Cl</smiles>

12c, $69 \%$<smiles>COc1ccc(-n2c(-c3ccccc3N)nc3ccccc3c2=N)cc1</smiles>

12e, $77 \%$<smiles>N=c1c2ccccc2nc(-c2ccccc2N)n1-c1ccccc1F</smiles>

12b, $79 \%$<smiles>N=c1c2ccccc2nc(-c2ccccc2N)n1-c1ccccc1Br</smiles>

12d, $73 \%$<smiles>N#Cc1ccccc1N</smiles>
o-Cyano anilines

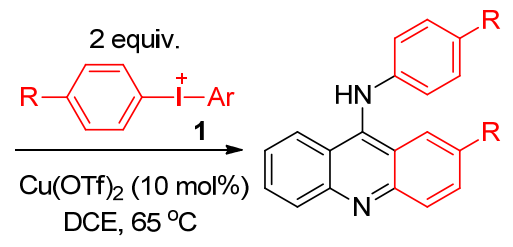

Acridines

13<smiles>c1ccc(Nc2c3ccccc3nc3ccccc23)cc1</smiles>

$13 a, 88 \%$<smiles>Clc1ccc(Nc2c3ccccc3nc3ccc(Cl)cc23)cc1</smiles>

13c, $84 \%$<smiles>COC(=O)c1ccc(Nc2c3cc(I)ccc3nc3ccc(I)cc23)cc1</smiles>

$13 e, 69 \%$<smiles>Cc1ccc(Nc2c3ccccc3nc3ccc(C)cc23)cc1</smiles>

13b, $92 \%$<smiles>Brc1ccc(Nc2c3ccccc3nc3ccc(Br)cc23)cc1</smiles>

13d, $90 \%$<smiles>Fc1cccc2nc3ccccc3c(Nc3ccccc3)c12</smiles>

13f, $69 \%$

图式 9 高价碘试剂与 $o$-氰基苯胺的亲电环化反应

Scheme 9 Electrophilic cyclization reactions between diaryliodonium salts and $o$-cyano anilines 


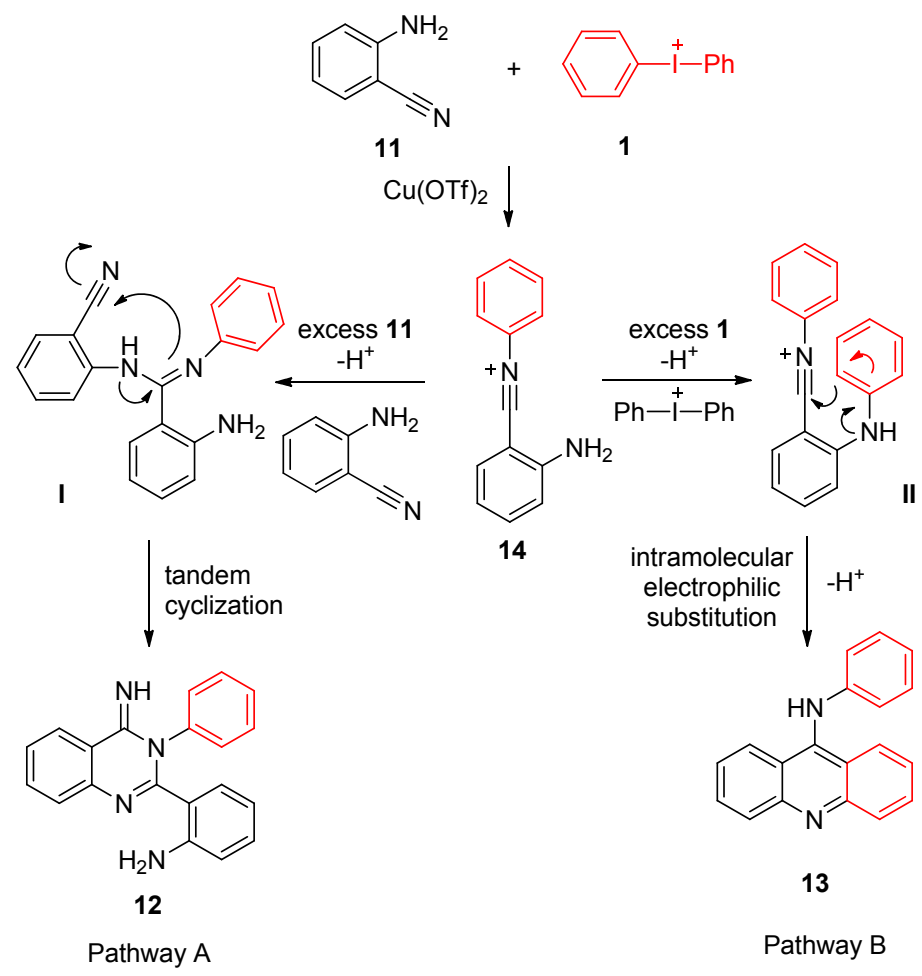

图式 10 高价碘试剂与 $o$-氰基苯胺的环化反应机理

Scheme 10 Proposed mechanism for diverse tandem cyclization reactions of $o$-cyanoanilines and diaryliodoniums.

提供了一些高效、实用的合成多取代复杂环化产物的途 径.

尽管对芳基高价碘盐诱导环化反应的研究报道并 不多, 但其在复杂环化产物合成领域已经表现出极大的 潜力和良好的发展前景, 更多的底物拓展及应用研究工 作正在进行.

\section{作者简介}

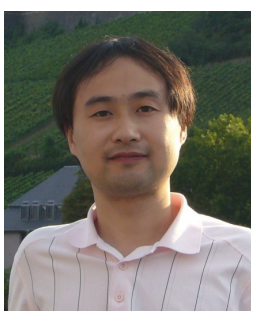

陈超, 1979 年 11 月生, 安徽芜湖人, 清华大学化学 系副教授. 2001 年清华大学化学系获学士学位, 2006 年 清华大学化学系获博士学位, 2007 年至 2011 年德国明 斯特大学博士后, 2011 年 5 月回到清华大学化学系担任 副教授. 主要研究方向为有机合成化学及元素有机化 学, 已发表 SCI 论文 40 余篇. 曾获德国洪堡基金会博士 后奖学金，亚洲前沿有机化学报告奖(ACP Lectureship), 清华大学学术新人奖, Thieme Chemistry Journal Award 等多项荣誉.

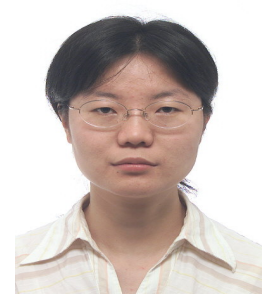

曲红梅, 1973 年 9 月生, 河北衡水人, 天津大学化 工学院副教授. 1996 年河北工业大学化工系获学士学 位, 1999 年天津大学应化系获硕士学位, 2003 年天津大 学化工学院获博士学位, 2005 年至 2007 年日本北海道 大学博士后, 2003 年至今, 天津大学化工学院任教. 主 要研究方向为有机合成化学, 已发表 SCI 论文 10 余篇. 曾获日本文部科学省博士后奖学金.

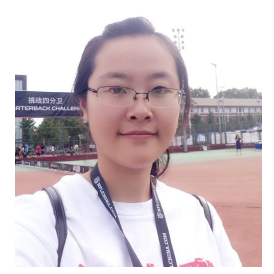

陈静，2012 年毕业于青岛科技大学化工学院，同年 进入天津大学化工学院制药工程系攻读硕士学位. 硕士 期间在清华大学化学系联合培养从事有机合成方法学 的研究. 


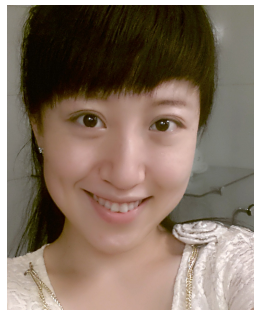

彭静, 2012 年毕业于清华大学化学系, 获得学士学 位, 同年进入清华大学化学系继续攻读博士学位, 主要 研究有机合成方法学.

\section{References}

[1] (a) Stang, P. J.; Zhdankin, V. V. J. Am. Chem. Soc. 1993, 115, 9808 .

(b) Boldyrev, A. I.; Zhdankin, V. V.; Simons, J.; Stang, P. J. J. Am. Chem. Soc. 1992, 114, 10569.

[2] For selected recent examples, see: (a) Fananas, M. M.; Feringa, B. L. J. Am. Chem. Soc. 2014, 136, 9894.

(b) Zhang, F.; Das, S.; Walkinshaw, A. J.; Casitas, A.; Taylor, M.; Suero, M. G.; Gaunt, M. J. J. Am. Chem. Soc. 2014, 136, 8851.

(c) Lindstedt, E.; Ghosh, R.; Olofsson, B. Org. Lett. 2013, 15, 6070.

(d) Castro, S.; Fernández, J. J.; Vicente, R.; Fañanás, F. J.; Rodríguez, F. Chem. Commun. 2012, 48, 9089.

(e) Hickman, A. J.; Sanford, M. S. ACS Catal. 2011, 1, 170.

(f) Chen, B.; Hou, X. L.; Li, Y. X.; Wu, Y. D. J. Am. Chem. Soc. 2011, 133, 7668.

(g) Wagner, A. M.; Sanford, M. S. Org. Lett. 2011, 13, 288.

(h) Xiao, B.; Fu, Y.; Xu, J.; Gong, T. J.; Dai, J. J.; Yi, J.; Liu, L. J. Am. Chem. Soc. 2010, 132, 468.

(i) Deprez, N. R.; Sanford, M. S. J. Am. Chem. Soc. 2009, 131, 11234.

(j) Xiao, Z. -C.; Xia, C.-F. Chin. J. Org. Chem. 2013, 33, 2119.

[3] (a) Merritt, E. A.; Olofsson, B. Angew. Chem., Int. Ed. 2009, 48, 9052 .

(b) Skucas, E.; MacMillan, D. W. C. J. Am. Chem. Soc. 2012, 134, 9090 .

(c) Bielawski, M.; Zhu, M.; Olofsson, B. Adv. Synth. Catal. 2007, 349, 2610.

(d) Bielawski, M.; Olofsson, B. Chem. Commun, 2007, 2521.

(e) Bielawski, M.; Olofsson, B. Org. Synth. 2009, 86, 308.

[4] For reviews on diaryliodonium salts, see: (a) Yusubov, M. S.; Maskaev, A. V.; Zhdankin, V. V. ARKIVOC 2011, (i), 370.

(b) Merritt, E. A.; Olofsson,B. Angew. Chem., Int. Ed. 2009, 48, 9052.

(c) Zhdankin, V. V.; Stang, P. J. Chem. Rev. 2008, 108, 5299.

(d)Zhdankin, V. V.; Stang, P. J. Chem. Rev. 2002, 102, 2523.

(e) Grushin, V. V. Chem. Soc. Rev. 2000, 29, 315.

(f) Stang, P. J.; Zhdankin, V. V. Chem. Rev. 1996, 96, 1123.

[5] Liu, C.; Zhang, W.; Dai, L.; You. S. Org. Lett. 2012. 14. 4525.

[6] (a) Culkin, D. A.; Hartwig, J. F. Acc. Chem. Res. 2003, 36, 234.

(b) Davies, H. M. L. Angew. Chem., Int. Ed. 2006, 45, 6422.

(c) Godula, K.; Sames, D. Science 2009, 312, 67.

(d) Doyle, M. P.; Duffy, R.; Ratnikov, M.; Zhou, L. Chem. Rev. 2010, 110, 704.

(e) Baudoin, O. Chem. Soc. Rev. 2011, 40, 4902.

(f) Sun, C.; Li, B.; Shi, Z. Chem. Rev. 2011, 111, 1293.

(g) Li, H.; Li, B.; Shi, Z. Catal. Sci. Technol. 2011, 1, 191.

(h) Wang, C.-Y. Synlett 2013, 24, 1606

[7] (a) Desai, L. V.; Hull, K. L.; Sanford, M. S. J. Am. Chem. Soc.
2004, 126, 9542 .

(b) Chen, X.; Goodhue, C. E.; Yu, J. J. Am. Chem. Soc. 2006, 128, 12634 .

(c) Shabashov, D.; Daugulis, O. J. Am. Chem. Soc. 2010, 132, 3965.

(d) Stowers, K. J.; Fortner, K. C.; Sanford, M. S. J. Am. Chem. Soc. 2011, 133, 6541.

(e) Qian, B.; Xie, P.; Xie, Y.; Huang, H. Org. Lett. 2011, 13, 2580.

(f) Nadres, E. T.; Daugulis, O. J. Am. Chem. Soc. 2012, 134, 7.

(g) Pan, F.; Shen, P.; Zhang, L.; Wang, X.; Shi, Z. Org. Lett. 2013, 15,4758 .

(h) Davies, H. M. L.; Beckwith, R. E. J. Chem. Rev. 2003, 103, 2861.

(i) Bhunia, S.; Liu, R. J. Am. Chem. Soc. 2008, 130, 16488.

(j) Horino, Y.; Yamamoto, T. Ueda, K.; Kuroda, S.; Toste, F. D. J. Am. Chem. Soc. 2009, 131, 2809.

[8] Peng, J.; Chen, C.; Chen, J.; Su, X.; Xi, C.; Chen, H. Org. Lett. 2014, 16, 3776.

[9] (a) Yang, Y.; Petersen, J. L.; Wang, K. J. Org. Chem. 2003, 68, 5832 .

(b) Camps, P.; Domingo, L. R.; Formosa, X.; Galdeano, C.; Gonález, D.; Munoz-Torrero, D.; Segalés, S.; Font-Bardia, M.; Solans, X. J. Org. Chem.2006, 71, 3464.

(c) Wu, Y. G.; Zhang, J. Y.; Bo, Z. S. Org. Lett. 2007, 9, 4435.

(d) Sánchez-Larios, E.; Holmes, J. M.; Daschner, C. L.; Gravel, M. Org. Lett. 2010, 12, 5772.

(e) Singh, C.; Hassam, M.; Naikade, N. K.; Verma, V. P.; Singh, A. S.; Puri, S. K. J. Med. Chem. 2010, 53, 7587.

[10] Chen, J.; Chen, C.; Chen, J.-J.; Wang, G.-H.; Qu, H.-M. Chem. Commun. 2015, 51, 1356

[11] (a) Boivin, T. L. B. Tetrahedron 1987, 43, 3309.

(b) Cardillo, G.; Orena, M. Tetrahedron 1990, 46, 3321

(c) Harding, K. E.; Tiner T. H., Comp. Org. Synth. 1991, 4, 363.

(d) Rousseau, G.; Homsi,F. Chem. Soc. Rev. 1997, $26,453$.

(e) Robin, S.; Rousseau, G. Tetrahedron 1998, 54, 13681.

(f) Robin, S.; Rousseau, G. Eur. J. Org. Chem. 2002, 3099.

(g) Ranganathan, S.; Muraleedharan, K.; Vaish M. N. K.; Jayaraman, N. Tetrahedron 2004, 60, 5273.

(h) French, A. N.; Bissmire, S.; Wirth, T. Chem. Soc. Rev. 2004, 33, 354.

(i) Tang, Y.; Oppenheimer, J.; Song, Z.; You, L.; Zhang, X.; Hsung, R. P. Tetrahedron 2006, 62, 10785.

(j) Montana, A. M.; Batalla, C.; Barcia, J. A. Curr. Org. Chem. 2009, 13, 919 .

[12] (a) Ernest, W.; Michelott, E. L.; Swindell, C. S.; Tingoli, M. J. Org. Chem. 1984, 49, 4894.

(b) Foster, A. B.; Jarman, M.; Leung, O.; Molague, R.; Leclercq, G.; Devleeschouwer, N. J. Med. Chem. 1985, 28, 1491.

(c) Wang, L.; Miller, L. O.; Nolte, R. T.; McDonnell, D. P.; Willson, T. M.; Zuercher, W. J.; Bioorg. Med. Chem. Lett. 2006, 16, 821.

[13] (a) Phipps, R. J.; McMurray, L.; Ritter, S.; Duong, H. A.; Gaunt, M. J. J. Am. Chem. Soc. 2012, 134, 10773.

(b) Chen, B.; Hou, X.; Li, Y.; Wu, Y. J. Am. Chem. Soc. 2011, 133, 7668 .

(c) Xiao, B.; Fu, Y.; Xu, J.; Gong, T.; Dai, J.; Yi, J.; Liu, L. J. Am. Chem. Soc. 2010, 132, 468.

(d) Phipps, R. J.; Gaunt, M. J. Science 2009, 323, 1593.

(e) Phipps, R. J.; Grimster, N. P.; Gaunt, M. J. J. Am. Chem. Soc. 2008, 130, 8172 .

[14] Selected recent examples: (a) Bhalla, V.; Vij, V.; Kumar, M.; Sharma, P. R.; Kaur, T. Org. Lett. 2012, 14, 1012. 
(b) Velusamy, M.; Chen, C. H.; Wen, Y. S.; Lin, J. T.; Lin, C. C.; Lai, C. H.; Chou, P. T. Organometallics 2010, 29, 3912.

(c) Li, H.; Jäkle, F. Macromolecules 2009, 42, 3448.

(d) Kim, J. L.; Shin, I. S.; Kim, H. J. Am. Chem. Soc. 2005, 127, 1614.

(e) Verhaeghe, P.; Azas, N.; Gasquet, M.; Hutter, S.; Ducros, C.; Laget, M.; Rault, S.; Rathelot P.; Vanelle, P. Bioorg. Med. Chem. Lett. 2008, 18, 396.

(f) Henderson, E. A.; Bavetsias, V.; Theti, D. S.; Wilson, S. C.; Clauss R.; Jackman, A. L. Bioorg. Med. Chem. 2006, 14, 5020.

(g) Chien, T. C.; Chen, C. S.; Yu, F. H.; Chern, J. W. Chem. Pharm. Bull. 2004, 52, 1422.

(h) Herget, T.; Freitag, M.; Morbitzer, M.; Kupfer, R.; Stamminger T.; Marschall, M. Antimicrob. Agents Chemother. 2004, 48, 4154.

[15] Wang, Y.; Chen, C.; Peng, J.; Li, M. Angew. Chem., Int. Ed. 2013, $52,5323$.

[16] Su, X.; Chen, C.; Wang, Y.; Chen, J.; Lou, Z.; Li, M. Chem. Commun. 2013, 49, 6752.

[17] (a)Xu, X.; Liu, Y.; Park, C. Angew. Chem., Int. Ed. 2012, 51, 9372. (b) Pin, F.; Comesse, S.; Sanselme, M.; Daïch, A. J. Org. Chem. 2008, 73, 1975.

(c) Du, W. Tetrahedron 2003, 59, 8649.

(d) Hutchinson, C. R. Tetrahedron 1981, 37, 1047.

(e) Zhao, L.; Xiong, F.; Chen, W.; Chen, F. Synthesis 2011, 4045.

(f) Anderson, R. J.; Raolji, G. B.; Kanazawa, A.; Greene, A. E. Org. Lett. 2005, 7, 2989.

(g) Liu, G.; Dong, Qi.; Yao, Y.; Yao, Z. Org. Lett. 2008, 10, 5393. (h) Wall, M. E.; Wani, M. C.; Cook, C. E.; Palmer, K. H.; MacPhail, A. T.; Sim, G. A. J. Am. Chem. Soc. 1966, 88, 3888.

(i) Kingsbury, W. D.; Boehm, J. C.; Jakas, D. R.; Holden, K. G.; Hecht, S. M.; Gallagher, G.; Caranfa, M. J.; McCabe, F. L.; Faucette, L. F.; Johnson, R. K.; Hertzberg, R. P. J. Med. Chem. 1991, 34,98 .

(j) Cragg, G. M.; Newman, D. J. J. Nat. Prod. 2004, 67, 232.

(k) Butler, M. S. Nat. Prod. Rep. 2005, 22, 162.

(1) Kaur, K.; Jain, M.; Reddy, R. P.; Jain, R. Eur. J. Med. Chem. 2010, 45, 3245.

[18] Wang, Y.; Chen, C.; Zhang, S.; Lou, Z.-B.; Su, X.; Wen, L.-R.; Li, M. Org. Lett. 2013, 15, 4794

[19] (a) Xu, H. H.; Wolf, C. Chem. Commun. 2009, 3035.

(b) Miyaura, N.; Suzuki, A. Chem. Rev. 1995, 95, 2457.

(c) Miyaura, N.; Suzuki, A. Chem. Commun. 1979, 866

[20] Chen, J. -J..; Chen, C.; Chen, J.; Gao, H.-P.; Qu, H.-M. Synlett 2014, 25, 2721.

[21] Pang, X.-L.; Chen, C.; Su, X.; Li, M. Org. Lett. 2014, 16, 6228.

[22] (a) Mark, W. J. Antimicrob Chemother. 2001, 47, 1.

(b) Srivastava A.; Nizamuddin Indian J. Heterocycl. Chem. 2004, 13, 261.

(c) Elslager, E. F.; Worth, D. F. J. Med. Chem. 1969, 12, 955.

(d) Pathak, R. B.; Bahel, S. C. J. Antibact. Antifunggl Agents 1980 , 8,15 .

(e) Takemura, Y.; Ju-ichi, M.; Ito, C.; Furukawa, H.; Tokuda, H.; Planta Med. 1995, 61, 366.

(f) Denny, W. A. Curr. Med. Chem. 2002, 9, 1655. 